University of Nebraska - Lincoln

DigitalCommons@University of Nebraska - Lincoln

October 2006

\title{
Remote bond breaking by interacting temporary anion states
}

\author{
Paul Burrow \\ pburrow1@unl.edu \\ Gordon A. Gallup \\ UNL,ggallup1@unl.edu
}

Follow this and additional works at: https://digitalcommons.unl.edu/physicsburrow

Part of the Physics Commons

Burrow, Paul and Gallup, Gordon A., "Remote bond breaking by interacting temporary anion states" (2006). Paul Burrow Publications. 31.

https://digitalcommons.unl.edu/physicsburrow/31

This Article is brought to you for free and open access by the Research Papers in Physics and Astronomy at DigitalCommons@University of Nebraska - Lincoln. It has been accepted for inclusion in Paul Burrow Publications by an authorized administrator of DigitalCommons@University of Nebraska - Lincoln. 


\title{
Remote bond breaking by interacting temporary anion states
}

\author{
P. D. Burrow ${ }^{\text {a) }}$ and G. A. Gallup ${ }^{\text {b) }}$ \\ Department of Physics and Astronomy, The University of Nebraska-Lincoln, Lincoln, Nebraska 68588-0111
}

(Received 12 July 2006; accepted 6 September 2006; published online 18 October 2006)

\begin{abstract}
The cross section for bond breaking at the site of a dissociative temporary negative ion state through the dissociative electron attachment process can be considerably enhanced by the presence of a second longer-lived temporary negative ion state elsewhere in the molecule, even one quite remote from the first. In a series of chloroalkenes possessing both $\mathrm{C}-\mathrm{Cl}$ and $\mathrm{C}=\mathrm{C}$ bonds separated by various distances, we show that the cross sections are determined by the lifetime of the lower anion state created by the mixing of the anion states of these two moieties, with the wave function's coefficients giving the probability that the electron is located at the dissociative site. Furthermore, the lifetime of the composite anion state can be expressed in terms of these same coefficients and the lifetimes of the unmixed resonances. We also discuss how these results may give insight into the means by which strand breaks are induced in DNA by the attachment of slow electrons. (C) 2006 American Institute of Physics. [DOI: 10.1063/1.2358683]
\end{abstract}

\section{INTRODUCTION}

Because bond breaking induced by low energy electrons is readily facilitated by the dissociative electron attachment (DEA) process

$$
e^{-}+A B \rightarrow A B^{-*} \rightarrow A+B^{-},
$$

the properties of the temporary negative ion intermediate largely dictate the efficiency for the reaction. In complex molecules, the temporary negative ion states are derived from those associated with each of the various functional groups. The mixing between these anion states, however, alters their energies and lifetimes from the values that would be observed in the separated moieties. An understanding of the properties of these coupled resonances in complex molecules is therefore essential. In particular, the influence of a long-lived nondissociative temporary anion state on the DEA process taking place at a site that is remote from the location of the original resonance is the topic of this present work.

Another of our goals is to gain insight into large molecular systems from a detailed analysis of a series of smaller molecules. It seems likely that there will continue to be molecules of biological interest that will exceed available computer capabilities and for which qualitative considerations are required for any understanding at all of the elementary processes involved. The molecules and methods used in this article are designed to improve our insight as well as to study the interesting phenomena of resonance interactions in low symmetry systems.

Strand breaks in DNA attributed to the DEA mechanism, ${ }^{1-4}$ provide an illustration of great radiological significance. The experimental evidence has been interpreted ${ }^{5}$ to suggest that temporary negative ion states as-

\footnotetext{
${ }^{a)}$ Electronic mail: pburrow1@unl.edu; URL: http://www.physics.unl.edu/ directory/burrow/Files/burrow.htm

${ }^{b)}$ Electronic mail: ggallup1@unl.edu; URL: http://www.unl.edu/ggallup/
}

sociated with the DNA bases transfer an electron to another site where a strand break may occur. Our purpose here is to view this sort of interaction in terms of quantum mechanical mixing of two temporary negative ion states and to determine how the altered resonance characteristics affect the physics of the remote bond breaking process.

In the present work, we consider the temporary negative ion states of a set of molecules

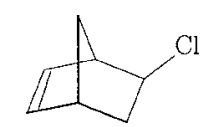

I

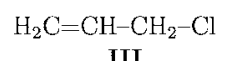

III

$\underset{\mathbf{V}}{\mathrm{H}_{2} \mathrm{C}=\mathrm{CH}-\left(\mathrm{CH}_{2}\right)_{3}-\mathrm{Cl}}$

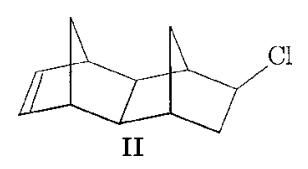

$\mathrm{H}_{2} \mathrm{C}=\mathrm{CHI}-\left(\mathrm{CH}_{2}\right)_{2}-\mathrm{Cl}$ IV

$\mathrm{H}_{2} \mathrm{C}=\mathrm{CH}-\left(\mathrm{CH}_{2}\right)_{4}-\mathrm{Cl}$
VI that are considerably simpler than the DNA nucleotides, but that preserve the essential features we wish to explore. In contrast to the nucleotides, substantial experimental data are available for these compounds, including their DEA cross sections and the vertical attachment energies (VAEs) required to place an electron into the normally unoccupied antibonding molecular orbitals. Each compound possesses two low-lying temporary anion states arising from mixing of a nominally ethylenic $\pi^{*}$ orbital on the $\mathrm{C}=\mathrm{C}$ bond and a nominally $\sigma^{*}$ orbital on the $\mathrm{C}-\mathrm{Cl}$ bond. (The asterisk indicates antibonding.) For convenience, the anion states created by the occupation of the individual $\pi^{*}$ and $\sigma^{*}$ orbitals, which are the ingredients of the observed resonances, will be called localized anion states (LAS). Compounds I and II, and III-VI allow us to explore the variation in the DEA cross section as a function of the separation between these two interacting functional groups. 

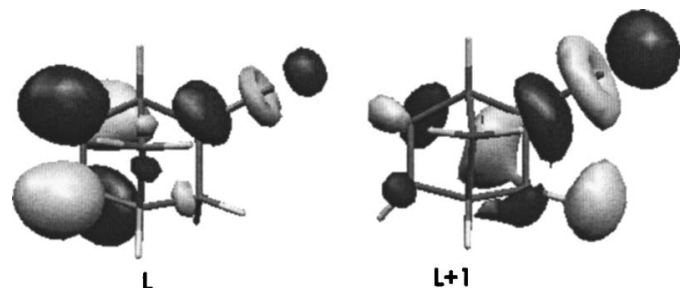

FIG. 1. LUMO and LUMO+1 for I.

Our procedure in brief is the following. After a discussion of the experimental data available for I-VI, we provide a simplified derivation of the widths (inverse lifetimes) of two coupled temporary negative ion states on moieties that are spatially separated from each other. We show that the widths can be expressed in terms of the wave function coefficients on each of the moieties and the widths characterizing the unmixed resonances, as they would appear in compounds containing only one or the other of the functional groups. Following this, we show a simple modification of the expression for the DEA cross section that incorporates these quantities and allows us to predict the DEA cross sections of I-VI. Next, molecular orbital calculations are carried out on I-VI in order to determine the mixing coefficients and, of considerable significance, the relative signs of terms consisting of the square roots of the widths of the unmixed resonances. Comparisons of the predicted DEA cross sections with experiment are then described. Finally, we discuss the implications of this analysis for strand breaks in DNA.

\section{BACKGROUND}

Compounds I-VI consist of an ethylenic group coupled through a saturated hydrocarbon bridge to a monochloroalkane. The first of these groups possesses a low-lying empty $\pi^{*}$ orbital, leading to a relatively long-lived temporary anion state with a minimum in the potential surface along the $\mathrm{C}=\mathrm{C}$ stretching coordinate. In ethylene itself, electron transmission spectroscopy ${ }^{6}$ (ETS) studies $^{7}$ show the existence of very faint vibrational structures appearing in the resonance peak in the total scattering cross section, indicating the nondissociative nature of this resonance along the $\mathrm{C}=\mathrm{C}$ stretch coordinate. The energy required to form this resonance in the geometry of the neutral molecule, that is, its VAE, is $1.76 \mathrm{eV}^{8}$

In monochloroalkanes, the lowest unoccupied molecular orbital (LUMO) lies along the $\mathrm{C}-\mathrm{Cl}$ bond and thus, locally, has $\sigma^{*}$ symmetry. The associated temporary anion state is short lived and dissociative, leading to the production of $\mathrm{Cl}^{-}$ by the DEA process. ${ }^{9}$ The VAEs of monochloroalkanes ${ }^{10-13}$ vary from 1.8 to $2.4 \mathrm{eV}$, excluding methyl chloride. Mixing of the $\pi^{*}$ and $\sigma^{*}$ orbitals in I-VI therefore leads to a LUMO that is primarily of $\pi^{*}$ character but one possessing an admixture of $\sigma^{*}$ that is a strong function of the separation between the two groups. The next higher empty orbital, $\mathrm{LUMO}+1$, of course, will commonly have the reverse distribution. We illustrate these orbitals for I in Fig. 1. ${ }^{14}$

The DEA cross sections of I and II have been measured by Pearl et al., ${ }^{15,16}$ and those of III-VI by UnderwoodLemons et al. ${ }^{17}$ and Aflatooni et al. ${ }^{18}$ Cross sections are also
TABLE I. Experimental DEA cross sections, peak energies, and VAEs for the chloroalkenes.

\begin{tabular}{lcccc}
\hline \hline Molecule & Expt. $\sigma_{\text {DEA }}\left(\mathrm{cm}^{2}\right)$ & Peak energy $(\mathrm{eV})$ & VAE $(\mathrm{eV})$ & Reference \\
\hline I & $4.9 \times 10^{-17}$ & 1.1 & $1.1^{\mathrm{a}}$ & 15 \\
II & $7.45 \times 10^{-18}$ & 1.35 & 1.26 & 16 \\
III & $2.10 \times 10^{-16}$ & 0.81 & 1.04 & 18 \\
& $2.38 \times 10^{-16}$ & 0.97 & $\ldots$ & 17 \\
& $1.99 \times 10^{-16}$ & 0.79 & 1.01 & 19 \\
IV & $5.70 \times 10^{-18}$ & 1.20 & 1.40 & 18 \\
& $1.48 \times 10^{-17}$ & 1.37 & $\ldots$ & 17 \\
V & $7.54 \times 10^{-19}$ & 1.24 & 1.58 & 18 \\
& $1.3 \times 10^{-18}$ & 1.30 & $\ldots$ & 17 \\
& $9.0 \times 10^{-19}$ & 1.50 & 1.6 & 19 \\
VI & $4.03 \times 10^{-19}$ & 1.23 & 1.68 & 18 \\
& $1.0 \times 10^{-18}$ & 1.26 & $\ldots$ & 17 \\
\hline
\end{tabular}

${ }^{\mathrm{a}}$ Reference 20.

reported for III and V by Modelli. ${ }^{19}$ The peak production of $\mathrm{Cl}^{-}$is found to occur very near the energy of the anion state associated with the LUMO, as determined by the location of the resonance appearing in the total scattering cross section, that is, the VAE. The magnitudes of the DEA cross sections are observed to decrease with increasing separation between the $\mathrm{C}=\mathrm{C}$ and $\mathrm{C}-\mathrm{Cl}$ moieties. Table I summarizes the peak DEA cross sections, the energies of the peaks and the VAEs for I-VI. In all the past studies, the mixing between the $\pi^{*}$ and $\sigma^{*}$ orbitals was noted, and the results were interpreted to suggest a picture in which capture of the electron occurs into the $\pi^{*}(\mathrm{C}=\mathrm{C})$ orbital and is subsequently transferred to the $\sigma^{*}(\mathrm{C}-\mathrm{Cl})$ orbital.

As Fig. 1 illustrates for I, the LUMO is, in fact, distributed over both ends of the molecule, with the greater portion residing on the $\mathrm{C}=\mathrm{C}$ bond. In all these compounds, it is therefore clear that attachment into the LUMO does not occur to the region of the $\pi^{*}$ orbital alone but, rather, the electron is delocalized to some extent on both ends. There are two additional aspects of this temporary anion state to note. First, the lifetime of this state is a property of its composite nature and will not be dictated solely by the short lifetime associated with the $\mathrm{C}-\mathrm{Cl} \sigma^{*}$ resonance or by the longer-lived $\pi^{*}$ resonance separately. The dominant contribution to the resonance width arises from the electron autodetachment rate in the elastic channel, and, initially, the charge density associated with the excess electron must decrease at the same rate at all points on the molecule. As the molecule distorts away from the geometry of the neutral, the decay width, of course, will change, as well as the relative coefficients on each moiety. Second, during this decay period the antibonding portion of the wave function on the $\mathrm{C}-\mathrm{Cl}$ moiety, even though initially small, exerts a force tending to stretch the $\mathrm{C}-\mathrm{Cl}$ bond, thus initiating the DEA process. The width of the composite $\pi^{*}-\sigma^{*}$ coupled resonance is therefore the key parameter, along with the fraction of the charge on the $\mathrm{C}-\mathrm{Cl}$ bond, that determines the DEA cross section in our model. We next present a derivation of this width in terms of the characteristics of two spatially separated resonances. 


\section{THEORY}

\section{A. A heuristic analysis of the widths of mixed temporary anion states}

\section{One shape resonance}

In cases where resonances occur in scattering, it is frequently convenient to think of their being superimposed upon a nonresonant background, which is then assumed to be substantially unvarying over the energy region of the resonance. We denote the background wave function by $\psi_{E}$, where its energy is shown explicitly. It should be noted that it is an $(n+1)$-electron function; that is, it could be given approximately by a Slater determinant with a number of doubly occupied molecular orbitals (DOMOs) representing the target molecule and a singly occupied molecular orbital (SOMO) representing the continuum function.

Now consider a target molecule that possesses one antibonding orbital $u^{*}$ that will show up as a shape resonance in the scattering cross section. We can construct an $(n+1)$-electron function in which we have the target molecule, again represented by the DOMOs as above, but now with $u^{*}$ as a SOMO. We denote this function by $\phi$. When $\phi$ and $\psi_{E}$ interact, we are led to a total wave function of the form

$$
\Psi_{E}=c_{E} \phi+\psi_{E},
$$

where $c_{E}$ is a coefficient to be determined. We use 1 for the $\psi_{E}$ coefficient, since the amplitude of this function is not too energy dependent and can be thought of as being determined, principally, by experimental conditions, such as the incident electron flux.

If we substitute $\Psi_{E}$ into Schrödinger's equation, we obtain a familiar $2 \times 2$ matrix eigenvalue problem in the usual way,

$$
\left[\begin{array}{cc}
w & v_{E} \\
v_{E} & E
\end{array}\right]\left[\begin{array}{c}
c_{E} \\
1
\end{array}\right]=\Lambda\left[\begin{array}{c}
c_{E} \\
1
\end{array}\right]
$$

where

$$
\begin{aligned}
& w=\langle\phi|H| \phi\rangle, \\
& E=\left\langle\psi_{E}|H| \psi_{E}\right\rangle,
\end{aligned}
$$

and

$$
v_{E}=\left\langle\psi_{E}|H| \phi\right\rangle,
$$

where $v_{E}$ could be called the lifetime amplitude. As we shall see, it is simply related to the width of the resonance $\Gamma$, in terms of which the lifetime is $\hbar / \Gamma$. For our present purposes the value of $\Lambda$ is of no particular interest.

We now assume that $E<w$ and solve the eigenvalue problem obtaining

$$
c_{E} \approx-\frac{v_{E}}{w-E},
$$

and we see that the magnitude of $c_{E} \phi$ grows as $E$ approaches the value of $w$ from below. A similar treatment with $E>w$ shows the same effect when $E$ approaches $w$ from above. Thus, the amount of $\phi$ in the total wave function rises relative to the background wave function as we approach $E=w$, but this heuristic treatment is too approximate to be carried through that point. The principal difficulty involves using equations in which there is only one value of $E$ in the background wave function. If a correctly averaged treatment were carried out, our expression for $c_{E}$ would not diverge at $E$ $=w$.

This, in effect, is what Fano ${ }^{21}$ did in his early treatment of resonances, and he arrived at the following expression:

$$
\left|c_{E}\right|^{2}=\frac{\Gamma / 2}{\pi\left[(w-E)^{2}+(\Gamma / 2)^{2}\right]},
$$

which is usually called the Breit-Wigner single level formula. It is useful, however, to compare the two results. When $|w-E| \gg \Gamma / 2$, the first term in an expansion of Eq. (7) is

$$
\left|c_{E}\right|^{2} \approx \frac{\Gamma}{2 \pi(w-E)^{2}},
$$

and comparing this with the other approximate expression for $\left|c_{E}\right|^{2}$ obtained from Eq. (6), we obtain the important formula

$$
\Gamma=2 \pi\left|v_{E}\right|^{2},
$$

which turns out to be exact. ${ }^{22}$ One can show that this result for $\Gamma$ is also a consequence of Fermi's "golden rule.",3

\section{Two shape resonances}

The results of Sec. III A 1 may be generalized to a larger number of resonances. In all of the molecules we treat, there is a $\pi^{*}$ antibonding orbital associated with a $\mathrm{C}=\mathrm{C}$ bond and a $\sigma^{*}$ antibonding orbital associated with a $\mathrm{C}-\mathrm{Cl}$ bond. Writing down the equations for the case of two resonances shows how the generalization goes. Under these circumstances the matrix corresponding to Eq. (2) is $3 \times 3$, and we have

$$
\left[\begin{array}{ccc}
w_{\pi \pi} & w_{\pi \sigma} & v_{\pi E} \\
w_{\sigma \pi} & w_{\sigma \sigma} & v_{\sigma E} \\
v_{E \pi} & v_{E \sigma} & E
\end{array}\right],
$$

where the subscripts refer to the two different antibonding orbitals, $u_{\pi}^{*}$ for the $\pi^{*}$ state and $u_{\sigma}^{*}$ for the $\sigma^{*}$ state. These lead to $(n+1)$-electron resonance functions $\phi_{\pi}$ and $\phi_{\sigma}$, respectively. We view these as "diabaticlike," since they are assumed to be localized at different places in the molecule and do not diagonalize the Hamiltonian directly. We also assume that they have properties that antibonding orbitals such as these would have if they were alone in a molecule.

We can diagonalize the above $3 \times 3$ matrix in two steps. The first eliminates the $w_{\pi \sigma}$ elements, and we obtain

$$
\left[\begin{array}{ccc}
w_{l} & 0 & \bar{v}_{l E} \\
0 & w_{u} & \bar{v}_{u E} \\
\bar{v}_{E l} & \bar{v}_{E u} & E
\end{array}\right],
$$

where we assume $w_{l}<w_{u}$, and the subscripts $l$ and $u$ stand for lower and upper resonances, respectively. The eigenfunction for the $w_{l}$ eigenvalue of the $2 \times 2$ sub-block of $H$ may now be written as 


$$
\phi_{l}=c_{\pi} \phi_{\pi}+c_{\sigma} \phi_{\sigma}
$$

with a corresponding value of

$$
\bar{v}_{l E}=c_{\pi} v_{\pi E}+c_{\sigma} v_{\sigma E}
$$

Similar expressions for $w_{u}$ could also be given, but we will not need these (see, however, the Appendix). As the second step, following the pattern of Eq. (1), we may now write the total wave function as

$$
\Psi=c_{l E} \phi_{l}+c_{u E} \phi_{u}+\psi_{E},
$$

and a perturbation treatment similar to that used above gives for $E<w_{l}$,

$$
\begin{gathered}
c_{l E} \approx-\frac{\bar{v}_{l E}}{w_{l}-E}, \\
c_{u E} \approx-\frac{\bar{v}_{u E}}{w_{u}-E} .
\end{gathered}
$$

Again, this shows how the electron probability density will be distributed as $E$ approaches the resonance from below.

We now obtain a $\Gamma$ as before and have

$$
\begin{aligned}
& \Gamma_{l E}=2 \pi\left|\bar{v}_{l E}\right|^{2} \\
& =\left|c_{\pi}\right|^{2} \Gamma_{\pi E}+4 \pi c_{\pi} c_{\sigma} v_{\pi E} v_{\sigma E}+\left|c_{\sigma}\right|^{2} \Gamma_{\sigma E},
\end{aligned}
$$

where $\Gamma_{\pi E}$ and $\Gamma_{\sigma E}$ are the widths the $\pi^{*}$ and $\sigma^{*}$ resonances would have, respectively, if they were alone in the molecule. Thus, Eq. (16) shows that the width of the lower resonance is related to the widths of the individual LASs.

In our later work we will use experimental values of the individual $\pi^{*}$ or $\sigma^{*}$ resonance widths, and from Eq. (9) we use the generic expression

$$
v=\sqrt{\frac{\Gamma}{2 \pi}}
$$

to obtain values of the $v$ 's for Eq. (16). The principal difficulty is that there is no a priori method for deciding upon the correct sign for the square root. We will detail our procedure for dealing with this problem in Sec. III B, but here we give the final expression that we use to connect the lifetime of the lower composite resonance with those of the individual LASs,

$$
\Gamma_{l E}=\left|c_{\pi} \sqrt{\Gamma_{\pi E}}+r c_{\sigma} \sqrt{\Gamma_{\sigma E}}\right|^{2},
$$

where $r$ is +1 or -1 depending on whether the relative signs of the square roots are the same or are different, respectively. We remind the reader of the significance of the terms. $c_{\pi}$ and $c_{\sigma}$ give the coefficients of the wave function for the $\mathrm{C}=\mathrm{C}$ bond and the $\mathrm{C}-\mathrm{Cl}$ bond, respectively. $\Gamma_{\pi E}$ and $\Gamma_{\sigma E}$ are the corresponding widths of the isolated resonances on these bonds. A key point to note is that the resonance width is actually a function of electron energy, $\Gamma=\Gamma(E)$, and all of these must be evaluated at the same energy, which is the energy of the lower composite anion state.

\section{Application to DEA}

Our objective in this article is to point at the physics leading to the enhanced DEA cross sections at remote sites that occur because of the coupling of the individual LASs. We will avoid the well known difficulties associated with $a b$ initio computations of this process by incorporating semiempirical values for those parameters that are most difficult to calculate. If there is but one resonance in a molecule and it is dissociative like $\mathrm{C}-\mathrm{Cl}$, a simple phenomenological expression proposed by Holstein ${ }^{24}$ for the DEA cross section can serve our purposes. He suggested that it be represented as the product of the capture cross section times a survival factor,

$$
\sigma_{\mathrm{DEA}}=\sigma_{\text {cap }} \exp \left(-\tau_{\mathrm{sep}} \Gamma / \hbar\right)
$$

where $\hbar / \Gamma$ is the lifetime of the electronic resonance and $\tau_{\text {sep }}$ is the separation time of the chemical bond. We will approximate the capture cross section by $\pi \lambda^{2}$ in the simplest cases. When, however, we have a molecule with two resonances that interact and only one is dissociative, only a fraction of the electron probability is associated with the antibonding orbital that causes the decomposition. Thus we expect the cross section to be reduced from what it would be if the "full force," so to speak, of the electron were present at the bond. We may obtain an idea of how this might affect the cross section quantitatively from an examination of the theoretical DEA formula derived by O'Malley. ${ }^{25,26}$ It is in the general form as Eq. (19), but with a more complicated expression for the capture cross section. Nevertheless, it contains the $\Gamma$ of the resonance associated with the dissociating bond in the numerator, and for our purposes we modify Eq. (19) to give

$$
\sigma_{\mathrm{DEA}}=\pi\left|c_{\sigma}\right|^{2} \lambda^{2} \exp \left(-\tau_{\mathrm{sep}} \Gamma_{l E} / \hbar\right)
$$

as our working equation, since only a relative amount, proportional to $\left|c_{\sigma}\right|^{2}$, of the electron is at the dissociative LAS. The preexponential factor is now the effective electron capture cross section as far as the dissociative bond is concerned. In Eq. (20) we have specialized our notation to match the previous theoretical sections. We wish to emphasize, however, how this expression will work in practice. The value of $\tau_{\text {sep }}$ is characteristic of the dissociating bond and, at the level of our approximation, will not depend on the nature of the second $\left(\pi^{*}\right)$ resonance in the molecule. In all of our cases this $\pi^{*}$ resonance is intrinsically lower in energy and has a longer lifetime (smaller $\Gamma$ ) than the $\sigma^{*}$ resonance, and the composite resonance function described in Eq. (10) will have the lower of the two experimental energies and will also have a longer lifetime than the $\sigma^{*}$ resonance, if it were alone in the molecule. Thus, although the DEA cross section is reduced by the $\left|c_{\sigma}\right|^{2}$ in front, the enhancement of lifetime more than compensates for this, and, generally, the presence of the $\pi^{*}$ resonance can dramatically increase the DEA cross section over its value in a similar molecule with only the $\sigma^{*}$ resonance present, as we will show. 


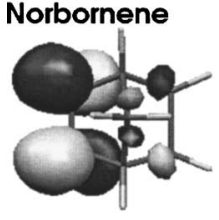

L

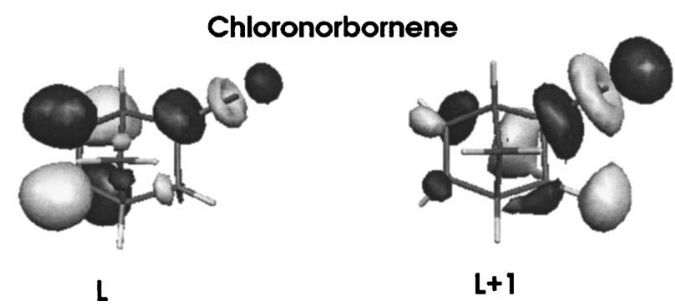

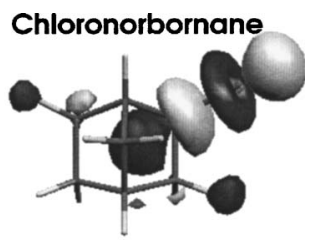

L

$L+1$
FIG. 2. I and the two modified versions, norbornene and 2-chloronorbornane, that are used to obtain the LASs. As discussed in the text, only the geometry for I is completely optimized.

\section{A sum rule for the resonance widths}

As a finish to this section we point out that a sum rule for resonance widths may be derived from our equations. If the two resonances we are dealing with are well isolated in energy from all others, one obtains

$$
\Gamma_{l E}+\Gamma_{u E}=\frac{\Gamma_{\pi E}+2 S_{\pi \sigma} r \sqrt{\Gamma_{\pi E} \Gamma_{\sigma E}}+\Gamma_{\sigma E}}{1-S_{\pi \sigma}^{2}},
$$

where $S_{\pi \sigma}$, defined in Sec. III B 2, is the overlap of the LASs and $r$ gives the relative signs of the square roots as described earlier. One sees that the sums of the widths are the same when the overlap is small enough to ignore. For the details the reader is referred to the Appendix.

\section{B. Methods used to obtain LASs and mixing coefficients}

\section{Determination of LASs}

To carry out our calculations we need specific functions for the $u_{\pi}^{*}$ and $u_{\sigma}^{*}$ antibonding orbitals of Sec. III A 2. We now outline our procedure for obtaining these, and we continue to use I to illustrate specifics. The calculations were all carried out with a $6-31 \mathrm{G}(d)$ basis using GAMESs. ${ }^{27}$

(1) The first step involved a full geometry optimization of I at the $6-31 \mathrm{G}(d)$ level. The $L$ and $L+1$ shown in the lower row of Fig. 2 are from this calculation.

(2) The geometry from step 1 was modified by replacing the $\mathrm{C}-\mathrm{Cl}$ bond with a $\mathrm{C}-\mathrm{H}$ bond at a conventional distance and the same angle. All other positions were unchanged. The LUMO for the norbornene (not quite geometry optimized) thus formed was determined, and this is shown in Fig. 2 (upper left).

(3) In a similar way the region in I around the $\mathrm{C}=\mathrm{C}$ bond was modified by movement of two $\mathrm{H}$ atoms and the inclusion of two more to give a molecule saturated in this vicinity. Again, no other atoms were moved. The LUMO for the 2-chloronorbornane thus formed was determined and is also shown in Fig. 2 (upper right).
For use in later calculations some modification (principally, movements of components to match atomic-orbital (AO) positions in molecule I) of the vectors was required. This, and the fact that the orbitals did not really come from I, necessitated their renormalization. In all of the cases we studied here, this renormalization was accomplished by multiplication with a constant in the range of 0.96-1.04. In the specific case of I the numbers are 0.983 for $u_{\pi}^{*}$ and 0.964 for $u_{\sigma}^{*}$, indicating that no significant loss of probability occurs in the transfer of these orbitals to the complete basis for I.

\section{The determination of $c_{\pi}, c_{\sigma}$, and the sign of $\sqrt{\Gamma}$}

As we saw in Sec. III A 2, the $2 \times 2$ block of the Hamiltonian matrix must be diagonalized first to produce the $c_{\pi}$ and $c_{\sigma}$ coefficients. We have discussed the generation of the LAS antibonding orbitals, and these are used as the SOMOs of the $(n+1)$-electron LAS functions $\phi_{\pi}$ and $\phi_{\sigma}$ that generate the $2 \times 2$ matrix we must diagonalize. Thus, the LAS functions will be mixed together to give lower and upper resonance states that will be closely equal to those that would have been obtained if the LUMO and LUMO +1 of the target molecule had originally been used as SOMOs for resonance functions. The difference is that our procedure gives the fractional mixing of the two individual resonances, a number that is crucial to the understanding of the influence of the $\pi^{*}$ resonance on DEA at the $\sigma^{*}$ resonance. One should also observe that our procedure does not produce an orthonormal basis for the $2 \times 2$ part of the Hamiltonian matrix, and, in general, the overlap between the two LAS functions is not zero, that is,

$$
S_{\pi \sigma}=\left\langle\phi_{\pi} \mid \phi_{\sigma}\right\rangle \neq 0,
$$

which provides a crude measure of the amount of interaction between the LASs.

The question of the signs of the $v$ 's arises because in calculations such as those described in Sec. III B 1 the orbitals produced by a program such as GAMESS have arbitrary signs. The $2 \times 2$ diagonalization described in the previous paragraph gives us correctly their signs relative to each other but not relative to the continuum function. From Eq. (5) we see that the arbitrary signs of the $u_{\pi}^{*}$ and $u_{\sigma}^{*}$ orbitals also affect the signs of the lifetime amplitudes $v_{\pi}$ and $v_{\sigma}$. It should be emphasized that these are simply off-diagonal matrix elements of a partially diagonalized Hamiltonian matrix, and examination of the values, if available, will give us the relative signs. ${ }^{27}$ We accomplished this by including in the basis of $(n+1)$-electron functions a set of functions in which the "continuum orbitals" are of the sort used in performing Stieltjes-imaging calculations. ${ }^{28,29}$ The functions were individual Gaussians consisting of $s, p_{x}, p_{y}$, and $p_{z}$ sets with different scale factors positioned at the molecule center of mass. When the Hamiltonian block associated with the pseudocontinuum functions is diagonalized, the $r$ in Eq. (18) is easily determined by inspection of the off-diagonal block. The various calculations needed to obtain all these quantities were carried out with a locally developed program, CRUNCH. ${ }^{30}$ 
TABLE II. Resonance mixing coefficients in I and II. The last column gives the relative phase of the lifetime amplitudes discussed in the text.

\begin{tabular}{lcrrr}
\hline \hline & $c_{\pi}$ & \multicolumn{1}{c}{$c_{\sigma}$} & \multicolumn{1}{c}{$S$} & \multicolumn{1}{c}{$r$} \\
\hline I & 0.956084 & -0.243967 & -0.056555 & 1 \\
II & 0.984538 & 0.162781 & 0.0130 .64 & -1 \\
\hline \hline
\end{tabular}

\section{CALCULATION OF PARAMETERS}

\section{A. Mixing of $\pi^{*}$ and $\sigma^{*}$ resonances in I and II}

Because of the rigid structures of I and II, the calculation of the interaction of the two resonances is straightforward, as described above, and we show the mixing coefficients in Table II along with the LAS overlaps $S$ and the relative phases of $v_{p}$ and $v_{s}$. We note that the overlaps behave as expected; the value corresponding to II is the smaller in magnitude.

\section{B. Mixing in III-VI}

Molecules III-VI, in contrast to I and II, are not rigid and occur in more than one conformation with a low-lying energy. Straight-chain chloroalkenes such as these, of general formula $\mathrm{C}_{n} \mathrm{H}_{(2 n-1)} \mathrm{Cl}$, have $n-2 \mathrm{C}-\mathrm{C}$ single bonds, and restricted Hartree-Fock (RHF) calculations at the 6-31G $(d)$ level, as were used here, predict that there are three local energy minima as rotation occurs around each bond. These minima appear roughly $120^{\circ}$ apart, and we expect $3^{(n-2)}$ rotamers for these compounds. For the molecules we have calculated, all but one of the rotamers are nonplanar and, hence, appear as enantiomeric pairs. It is likely that a similar situation holds with longer straight-chain chloroalkenes, and the number of distinct rotamers is expected to be

$$
\frac{3^{(n-2)}+1}{2}
$$

Thus, the distinct population numbers for III-VI are 2, 5, 14, and 41 , respectively. Underwood-Lemons et al. ${ }^{17}$ have calculated the rotamer energies for IV at the $6-31 \mathrm{G}(d)$ level, and we confirm their results. The different conformations of IV-VI require separate calculations for each of the rotamers that will be populated at the temperature of the experimental measurements $\left(\approx 60{ }^{\circ} \mathrm{C}\right)$ The seven lowest energy rotamers for $\mathrm{V}$ have been calculated. These comprise $91.1 \%$ of the population. We have also calculated mixing coefficients for what appear likely to be the two most prevalent rotamers of VI; we will discuss these results later. The mixing coefficients for III-VI are shown in Table III.

In Fig. 2 we showed an example of the antibonding orbitals in I and the modified molecules used in calculating the LAS orbitals. For comparison Fig. 3 shows corresponding drawings for compound $\mathrm{V}$. We note that the electron amplitude on the $\mathrm{C}-\mathrm{Cl} \mathrm{LAS}$ is not apparent with the existing plotting software.
TABLE III. Resonance mixing coefficients in III-VI.

\begin{tabular}{llllll}
\hline \hline & \multicolumn{1}{c}{$c_{\pi}$} & \multicolumn{1}{c}{$c_{\sigma}$} & \multicolumn{1}{c}{$s$} & \multicolumn{1}{c}{ Population } \\
\hline III & 0.831474 & -0.393863 & -0.234397 & 1 & 0.95 \\
& 1.0 & 0.0 & $0.0^{\mathrm{a}}$ & 1 & 0.05 \\
IV & 0.834276 & -0.343276 & -0.324989 & -1 & 0.563 \\
& 0.956785 & -0.123078 & -0.294730 & -1 & 0.234 \\
& 0.905226 & 0.280176 & 0.201219 & 1 & 0.117 \\
& 1.0 & 0.0 & $0.0^{\mathrm{a}}$ & 1 & 0.076 \\
& 0.997770 & -0.174212 & 0.074130 & -1 & 0.010 \\
$\mathrm{~V}$ & & & & \\
& -0.966587 & 0.109149 & -0.254953 & -1 & 0.210 \\
& 1.002969 & 0.092254 & -0.078126 & -1 & 0.199 \\
& 0.962056 & 0.156062 & 0.166822 & 1 & 0.179 \\
& 0.999232 & 0.012304 & 0.056288 & 1 & 0.134 \\
& 0.994776 & 0.028492 & 0.169503 & 1 & 0.123 \\
& 1.0 & 0.0 & $0.0^{\mathrm{a}}$ & 1 & 0.024 \\
& 0.910095 & 0.273405 & 0.194868 & 1 & 0.042 \\
VI & 0.967322 & 0.158921 & 0.126953 & 1 & 0.663 \\
& -0.924548 & 0.254824 & -0.179369 & -1 & 0.337 \\
\hline \hline
\end{tabular}

$\overline{\bar{a}}$ These rotamers are planar with $C_{s}$ symmetry. Thus the $\sigma-\pi$ designations are equivalent to $A^{\prime}-A^{\prime \prime}$, and the two LASs are exactly orthogonal.

\section{Widths of the LAS resonances}

\section{The $\sigma^{*}$ resonance}

The shape resonances associated with the empty $\mathrm{C}-\mathrm{Cl}$ MO have been observed in a number of monochloroalkanes by ETS. ${ }^{10-13}$ The widths of these features as they appear in the derivatives of the total scattering cross section increase monotonically with VAE in a manner consistent with the attachment of a $p$-wave electron. ${ }^{13}$ From such a plot, the energy separation of the dip and peak, $\Delta_{d p}$, in the derivative signal may be found for a particular VAE. Assuming that the background scattering does not interfere, $\Delta_{d p}$ is related to the $\Gamma$ appearing in the Breit-Wigner formula [Eq. (7)] by

$$
\Gamma=\sqrt{3} \Delta_{d p} .
$$

The chloronorbornane resonance appears at VAE $=2.30 \mathrm{eV} ;{ }^{20}$ however, these authors did not report the $\Delta_{d p}$ for this feature. Using the general dependence of $\Delta$ upon VAE observed in Ref. 13, we infer the value $\Delta_{d p}=1.7 \mathrm{eV}$ and the corresponding $\Gamma=2.94 \mathrm{eV}$ at the VAE.

For ethyl chloride, used to model the $\sigma^{*}$ resonances in
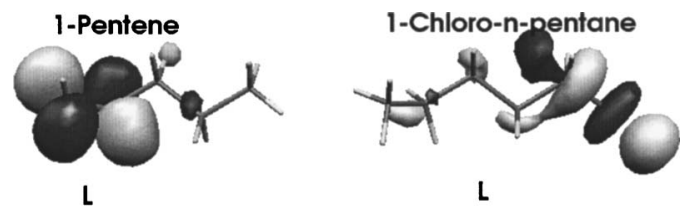

$\mathbf{L}$

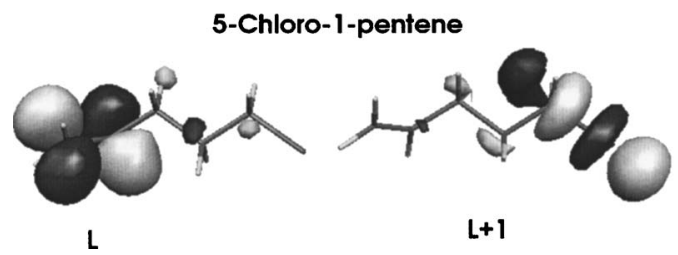

FIG. 3. V and the two molecules with modified atoms and positions used to calculate the LASs. 
III-VI, the VAE lies at $2.41 \mathrm{eV}$ with $\Delta_{d p}=1.72 \mathrm{eV},{ }^{13}$ yielding a $\Gamma_{\sigma}=2.98 \mathrm{eV}$. As discussed elsewhere, ${ }^{13}$ the contribution of Franck-Condon effects to $\Delta_{d p}$ for resonances of such short lifetimes can be ignored.

As noted earlier, the application of our key result [Eq. (18)] to determine $\Gamma$ for the composite anion state requires evaluation of $\Gamma_{\sigma E}$ and $\Gamma_{\pi E}$ at the energy of this resonance, rather than at the respective VAEs of the LASs. For this purpose we invoke the threshold law energy dependence,

$$
\Gamma \propto E^{l+1 / 2}, \quad E \rightarrow 0,
$$

anchoring the expression at the experimental $\Gamma$ evaluated by experiment at the VAE. For the $\sigma^{*}$ resonance the dependence is given by $E^{1.5}$ for a pure $p$ wave. ETS measurements ${ }^{13}$ found $E^{1.4}$ in close agreement.

\section{The $\pi^{*}$ resonances}

Obtaining an experimental estimate for the $\pi^{*}$ resonance widths in norbornene and the 1 -alkenes is less certain. The ET spectrum of ethylene shows an overall $\Delta_{d p} \approx 0.8 \mathrm{eV}$, corresponding to $\Gamma_{\pi} \approx 1.39 \mathrm{eV}$. However, it also displays weak evidence of nuclear motion ${ }^{7}$ during the lifetime of the anion, with three levels evident and spaced by $165 \pm 10 \mathrm{meV}$. These features are characteristic of the $\mathrm{C}=\mathrm{C}$ stretching mode excited by the presence of the antibonding electron. Clearly the Franck-Condon effects contribute to the apparent overall width. If the two upper vibrational structures are shifted to lie on top of the lowest feature, $\Delta_{d p}$ would be reduced by $\approx 2 \times 0.165=0.33 \mathrm{eV}$, yielding an estimate of $\Delta_{d p}$ $\approx 0.47 \mathrm{eV}$ arising from the finite electronic state lifetime. This corresponds to $\Gamma_{\pi} \approx 0.8 \mathrm{eV}$. Thus, the estimated range for ethylene is $0.8 \leqslant \Gamma_{\pi} \leqslant 1.39 \mathrm{eV}$. Because of the reduced symmetry of the double bond in norbornene and the 1-alkenes, we expect that $\Gamma_{\pi}$ in these compounds may be somewhat larger than that of ethylene. Consequently, we favor a value nearer the upper bound of the range.

We will examine later the effect of variations in this choice of $\Gamma_{\pi}$ on our calculated DEA cross sections. As above for the $\sigma^{*}$ resonances, a threshold energy dependence given by Eq. (22) is assumed, with $l=2$ for the $\pi^{*}$ LAS.

\section{COMPUTATION OF DEA CROSS SECTIONS}

\section{A. Average separation time for $\mathrm{C}-\mathrm{Cl}$ bond}

With expressions for the $\Gamma$ s and wave function amplitudes of the LUMOs in terms of known quantities for the separated LAS components, we are now ready to predict the cross sections arising from coupling of the two resonances. We propose to use Eq. (20) for this purpose; however, there is one quantity as yet undetermined, namely, $\tau_{\text {sep. }}$ Since this is a characteristic of the $\mathrm{C}-\mathrm{Cl}$ bond appearing in all of our compounds, we obtain it by fitting Eq. (19) to the experimental results in chloronorbornane for use with I and II. The measured peak value of the DEA cross section is $\sigma_{\mathrm{DEA}}$ $=6.9 \times 10^{-19} \mathrm{~cm}^{2}$ at $1.7 \mathrm{eV}^{15}$ As discussed in Sec. IV C 1 the value for $\Gamma_{\sigma}$ is $2.94 \mathrm{eV}$. We approximate the capture cross section by $\pi \lambda^{2}$, and at $1.7 \mathrm{eV}$ one obtains 7.04 $\times 10^{-16} \mathrm{~cm}^{2}$ for its value. Thus $\tau_{\text {sep }}$, the average separation time in the survival factor, must be $2.24 \times 10^{-16} \mathrm{~s}$. This may be expressed another way by noting that $\hbar / \tau_{\text {sep }}=0.4244 \mathrm{eV}$. For convenience in calculations we write Eq. (19) as

$$
\sigma_{\mathrm{DEA}}=c_{\text {cap }} \exp (-K \Gamma)
$$

where $K=2.356(\mathrm{eV})^{-1}$ for chloronorbornane. A check on this value may be obtained by consideration of 2-chloropropane, which is also a secondary chloride. Using data in Ref. 11, a similar treatment yields $K=2.40 \mathrm{eV}^{-1}$.

For compounds III-VI we fit to experimental results in ethyl chloride and find $K=2.870 \mathrm{eV}^{-1}$. Similarly, with 1-chloropropane we find $K=2.84 \mathrm{eV}^{-1}$ in close agreement. These exercises indicate the importance of emulating as closely as possible the characteristics of the group being modeled.

\section{B. Predicted DEA cross sections}

In Table IV, we list the computed values of $\Gamma_{\pi E}$ and $\Gamma_{\sigma E}$ for each of the rotamers of I-VI evaluated at the measured energies of the respective DEA peaks listed in Table I for these molecules. As described above, these are determined by use of the threshold energy dependence of resonances in the LASs and the anchor points given by the estimated values of $\Gamma_{\pi}$ and $\Gamma_{\sigma}$ in the reference LASs. In comparing our calculated cross sections with experiment, we found that the best least squares fit for $\mathrm{I}-\mathrm{V}$ was obtained with $\Gamma_{\pi}$ $=1.40 \mathrm{eV}$, just at the upper range of the values estimated for ethylene. All of the results in Table IV reflect this value.

The column labeled $\Gamma_{l E}$ shows the computed composite resonance width determined with Eq. (18). The DEA peak cross section for each rotamer is shown as $\sigma_{\mathrm{DEA}}$, and next to it is its population-weighted contribution to the total cross section. The entries labeled "Tot." show the summed values, that is, the predicted total cross section for each compound. The rightmost column gives, for comparison, the measured cross sections from Refs. 15 and 16 for I and II, respectively, and from Ref. 18 for III-IV, which we prefer for reasons of experimental consistency with the data obtained for I and II.

In Fig. 4 we plot the calculated DEA cross sections against the measured ones on a log-log scale. Data in perfect agreement would lie on the straight line of unit slope. The predicted cross section for VI is subject to more error than the others because only two of the rotamers are included out of the 41 possible.

Agreement with experiment is excellent for the rigid compounds I and II and for III which has only one important conformer. The molecules with multiple rotamers fair less well, with IV lying a factor of 2 below experiment and $\mathrm{V}$ a factor of 3 above. These errors may arise from inaccuracies in calculating rotamer energies at the self-consistent field (SCF) level, as well as from a need to thermally average the molecules more accurately around their local minima.

Nevertheless, considering that the cross sections span a range in excess of $10^{2}$, we argue that the essential physics of the interaction of two LASs has been captured in our present approach. 
TABLE IV. Results for individual rotamers.

\begin{tabular}{|c|c|c|c|c|c|c|}
\hline & $\begin{array}{l}\Gamma_{\pi E} \\
(\mathrm{eV})\end{array}$ & $\begin{array}{l}\Gamma_{\sigma E} \\
(\mathrm{eV})\end{array}$ & $\begin{array}{c}\Gamma_{l} \\
(\mathrm{eV})\end{array}$ & $\begin{array}{l}\sigma_{\mathrm{DEA}} \\
\left(\mathrm{cm}^{2}\right)\end{array}$ & $\begin{array}{c}\operatorname{pop} \times \sigma_{\text {DEA }} \\
\left(\mathrm{cm}^{2}\right)\end{array}$ & $\begin{array}{l}\text { Expt. } \\
\left(\mathrm{cm}^{2}\right)\end{array}$ \\
\hline I & 0.439 & 1.530 & 0.101 & $5.10 \times 10^{-17}$ & $5.10 \times 10^{-17}$ & \\
\hline Tot. & & & & & $5.10 \times 10^{-17}$ & $4.90 \times 10^{-17}$ \\
\hline II & 0.732 & 2.081 & 0.348 & $1.04 \times 10^{-17}$ & $1.04 \times 10^{-17}$ & \\
\hline Tot. & & & & & $1.04 \times 10^{-17}$ & $7.50 \times 10^{-18}$ \\
\hline \multirow[t]{2}{*}{ III } & 0.204 & 1.126 & 0.003 & $2.28 \times 10^{-16}$ & $2.16 \times 10^{-16}$ & \\
\hline & 0.204 & 1.126 & 0.196 & 0.0 & 0.0 & \\
\hline Tot. & & & & & $2.16 \times 10^{-16}$ & $2.10 \times 10^{-16}$ \\
\hline \multirow[t]{5}{*}{ IV } & 0.545 & 2.030 & 1.193 & $3.83 \times 10^{-18}$ & $2.16 \times 10^{-18}$ & \\
\hline & 0.545 & 2.030 & 0.752 & $1.75 \times 10^{-18}$ & $4.09 \times 10^{-19}$ & \\
\hline & 0.545 & 2.030 & 1.110 & $3.24 \times 10^{-18}$ & $3.79 \times 10^{-19}$ & \\
\hline & 0.545 & 2.030 & 0.523 & 0.0 & 0.0 & \\
\hline & 0.545 & 2.030 & 0.940 & $2.04 \times 10^{-18}$ & $2.04 \times 10^{-20}$ & \\
\hline Tot. & & & & & $2.97 \times 10^{-18}$ & $5.70 \times 10^{-18}$ \\
\hline \multirow[t]{7}{*}{ V } & 0.592 & 2.132 & 0.728 & $8.00 \times 10^{-19}$ & $1.68 \times 10^{-19}$ & \\
\hline & 0.592 & 2.132 & 0.301 & $8.20 \times 10^{-18}$ & $1.63 \times 10^{-18}$ & \\
\hline & 0.592 & 2.132 & 0.907 & $1.74 \times 10^{-18}$ & $3.12 \times 10^{-19}$ & \\
\hline & 0.592 & 2.132 & 0.594 & $2.66 \times 10^{-20}$ & $3.58 \times 10^{-21}$ & \\
\hline & 0.592 & 2.132 & 0.625 & $1.30 \times 10^{-19}$ & $1.60 \times 10^{-20}$ & \\
\hline & 0.592 & 2.132 & 0.567 & 0.0 & 0.0 & \\
\hline & 0.592 & 2.132 & 1.176 & $2.47 \times 10^{-18}$ & $1.03 \times 10^{-19}$ & \\
\hline Tot. & & & & & $2.23 \times 10^{-18}$ & $7.50 \times 10^{-19}$ \\
\hline \multirow[t]{2}{*}{ VI } & 0.580 & 2.107 & 0.906 & $1.83 \times 10^{-18}$ & $1.21 \times 10^{-18}$ & \\
\hline & 0.580 & 2.107 & 1.122 & $2.53 \times 10^{-18}$ & $8.52 \times 10^{-19}$ & \\
\hline Tot. & & & & & $2.06 \times 10^{-18}$ & $4.00 \times 10^{-19}$ \\
\hline
\end{tabular}

\section{Allyl chloride}

Because of the close proximity of the $\mathrm{C}=\mathrm{C}$ and $\mathrm{C}-\mathrm{Cl}$ groups in III, allyl chloride, this compound presents an important test case of the separated LASs. Furthermore, the computed value of $\Gamma_{l E}$ shown in Table IV is strikingly small, and it is useful to attempt to understand why this occurs. Indeed, substantial reductions are computed for this parameter in I and II, although not to such an extreme.

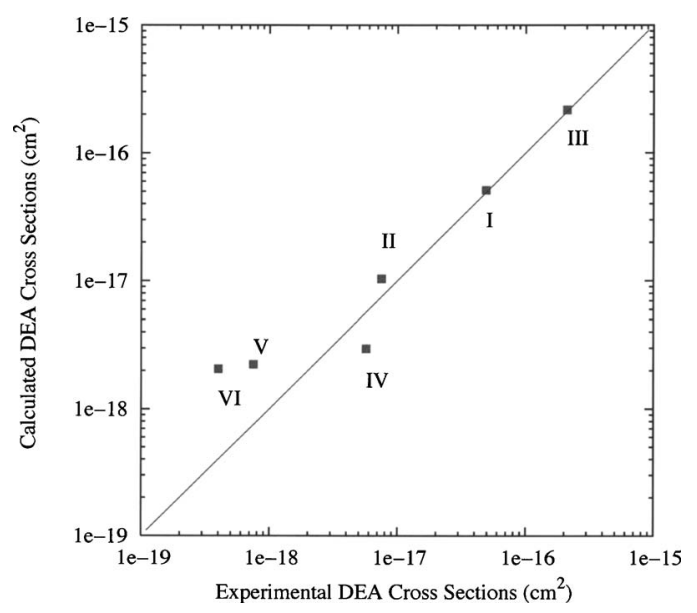

FIG. 4. A comparison of calculated and experimental DEA cross sections. The calculated values utilize $\Gamma_{\pi}=1.40 \mathrm{eV}$, the width obtained from a least squares fit of the data for I-V. The point for VI is also shown, but is not included in the minimization.
Figure 5 shows the LUMO, the LUMO+1, and the two LASs for allyl chloride. Mathematically, the small value of $\Gamma_{l E}$ can be traced to the negative value of $r$ appearing in Eq. (18), which, in turn, arises from the way each LAS is coupled to the continuum. In effect, the combination of the wave functions tends to reduce the lower $l$-value components
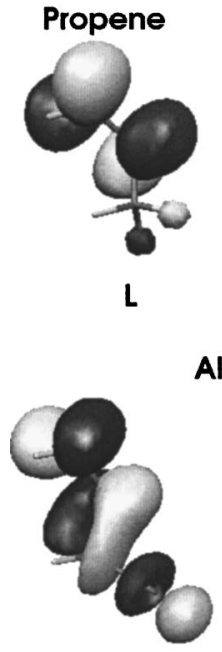

L

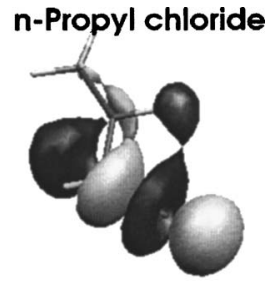

L
Allyl chloride

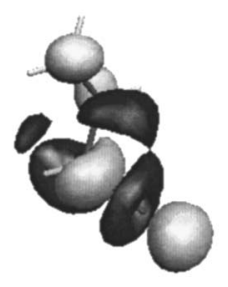

$L+1$

FIG. 5. Orbital drawings of the two LASs, the LUMO, and the LUMO+1 of allyl chloride. 
TABLE V. Populations $P_{l m}$ of $l$ and $m$ components of the allyl chloride LUMO and its two LASs for $l=0,1$, and 2 .

\begin{tabular}{lrrrr}
\hline \hline$l$ & $m$ & LUMO & $\pi^{*}$ LAS & $\sigma^{*}$ LAS \\
\hline 0 & 0 & 0.0119 & 0.0010 & 0.3167 \\
1 & -1 & 0.0070 & 0.0002 & 0.0117 \\
& 0 & 0.2268 & 0.3471 & 0.0304 \\
& 1 & 0.0024 & 0.0000 & 0.0792 \\
& & & & \\
& -2 & 0.0064 & 0.0000 & 0.0789 \\
& -1 & 0.2050 & 0.3232 & 0.0217 \\
& 0 & 0.0024 & 0.0019 & 0.0131 \\
& 1 & 0.0080 & 0.0001 & 0.0261 \\
& 2 & 0.0032 & 0.0011 & 0.0093 \\
\hline \hline
\end{tabular}

of the LUMO and yields a small $\Gamma_{l E}$ at the energy of the DEA peak. Since this VAE is relatively low, one observes a much reduced width.

In order to demonstrate this numerically, we have expanded the LUMO and LAS orbitals in spherical harmonics about the center of mass of the molecule. For each $l$ and $m$ pair this yields a radial function $R_{l m}(r)$, and we may define a population for each with the integral,

$$
P_{l m}=\int_{0}^{\infty} r^{2} d r\left|R_{l m}(r)\right|^{2} .
$$

Values of $P_{l m}$ are shown for low $l$ values in Table V, and it seen that for these $l$ values there is a tendency for the population of a particular $l-m$ state to be lower in the composite LUMO of the molecule than in the $\pi^{*}$ LAS. Higher $l$ values are relatively unimportant at the low energies involved. Thus the width is much reduced.

A more qualitative description of this phenomenon is possible. Examination of Fig. 5 shows that there are at least five nodal surfaces in the LUMO, and it changes sign fairly rapidly from point to point as position changes. From Table I we see that the DEA peak energy is $0.81 \mathrm{eV}$, and at this low energy we expect the continuum function to be slowly changing. Thus, considerable cancellation is possible in the matrix element giving the lifetime amplitude. The details of this depend, of course, on the angle between the two LASs and the relative magnitudes of the positive and negative portions of the orbital. The outcome of all of these contributions is a relatively long lifetime for this resonance.

\section{IMPLICATIONS FOR BOND BREAKING IN DNA}

The general features of the treatment above are applicable to more complex systems such as the nucleotides of DNA and ultimately to short strands of DNA itself. In these complex systems, the DNA bases provide low-lying temporary anion states arising from occupation of empty $\pi^{*}$ orbitals $^{31}$ analogous to the ethylenic group in the present study. Occupation of empty $\sigma^{*}$ orbitals at a number of possible sites will lead to repulsive anion states that produce fragmentation if the products have sufficiently high electron affinities, paralleling the action of the $\mathrm{C}-\mathrm{Cl}$ group in this study. Although a good deal is now known about the $\pi^{*}$ resonances of the bases, the energies of shape resonances in the remainder of the nucleotide, that is, the sugar ring and phosphate group, and reliable DEA cross sections associated with these anion states are not yet available. Nevertheless, calculations of the lowest few empty MOs in the systems of choice will give considerable insight into the mixing of the base $\pi^{*}$ orbitals with remote $\sigma^{*}$ bearing groups.

Theoretical studies by Bendys et al. have shown that an electron placed in a $\pi^{*}$ orbital on a DNA base can migrate through the adjacent deoxyribose to a $\sigma^{*}$ orbital at the $3^{\prime}$ $\mathrm{C}-\mathrm{O}$ site as the $\mathrm{C}-\mathrm{O}$ bond is stretched. This provides the basis for their studies of bond breaking in nucleotides, and it is useful to contrast their treatment with that in the present work. The principal features in the briefest form are the following: The initial electron attachment is viewed as occurring to the base $\pi^{*}$ orbital alone, rather than to the delocalized LUMO of the system. Coupling between the $\pi^{*}$ and relevant $\sigma^{*}$ orbital is calculated to be sufficiently large that the rate of transport of the electron through the intermediate bonds to the $\sigma^{*}$ orbital is not a rate limiting step. As a function of the $\mathrm{C}-\mathrm{O}$ bond stretch, there is a barrier in the adiabatic anion surface, owing to the avoided crossing of the $\pi^{*}$ and $\sigma^{*}$ anion states, and the thermal population of the associated vibrational mode is relied on to produce molecules with energies that can reach the $\mathrm{C}-\mathrm{O}$ bond length at the barrier. An electron attaching in the base $\pi^{*}$ orbital at this time can promptly move through bonds to the $\mathrm{C}-\mathrm{O} \sigma^{*}$, leading to dissociation. A key aspect of their model is that the phosphate group is assumed to be protonated and thus is charge neutral. As a consequence, the phosphate fragment has a high electron affinity, and in this regard it serves as the analog to chlorine in the present work.

The rates for the thermally activated process are in competition with the lifetime of the initially formed $\pi^{*}$ anion state on the base. Not surprisingly, the anion barrier height at the crossing is sensitive to the location of the $\pi^{*}$ resonance. In the model of Berdys et al., the $\pi^{*}$ energy is moved rather artificially over a range of energies to simulate the width of the lowest $\pi^{*}$ resonance. The bond breaking rate decreases rapidly as the $\pi^{*}$ energy is lowered. It would thus appear that the higher-lying base $\pi^{*}$ anion states ${ }^{31}$ are much more likely to contribute than the lowest.

Based on the present work, we suggest a different scenario, namely, that electron attachment occurs into an empty MO, which is delocalized over the base $\pi^{*}$ and other $\sigma^{*}$ orbitals, and that the fractional electron population on the $3^{\prime}$ $\mathrm{C}-\mathrm{O}$ bond, or on other strongly antibonding $\sigma^{*}$ sites, initiates bond stretching, without the necessity of a thermally excited vibrational mode. There is no doubt that such a population may enhance the DEA process, as is well known from temperature dependence studies in numerous molecules, but it is not required in our mechanism, nor is it invoked in the present calculations. As we have shown, mixing of the $\pi^{*}$ and $\sigma^{*}$ resonances alters the lifetime of the composite resonance in a way that enhances the DEA process, and the maximum in the cross section is expected to lie near the $\pi^{*}$ resonance energy. In a similar fashion, occupation of the other $\pi^{*}$ orbitals of the bases, and the $\sigma^{*}$ orbitals to which they are coupled, will give rise to other peaks in the DEA cross section at the energies of the base $\pi^{*}$ resonances. We note that 
coupling of the $3^{\prime} \mathrm{C}-\mathrm{O} \sigma^{*}$ orbital with base $\pi^{*}$ orbitals takes place through three saturated bonds in the nucleotides. The longer of our two rigid compounds, II, shows a considerable enhancement even through four saturated bonds.

Finally, we note that electron beam measurements of $\mathrm{Cl}^{-}$ yield from chloroalkanes show a peak at the nominal zero energy of the electron beam, in addition to the main peak associated with vertical attachment into the $\mathrm{C}-\mathrm{Cl} \sigma^{*}$ orbital $^{13}$ The "zero peak" is well known to arise from electron attachment to vibrationally excited levels of the neutral molecule whose energies are near the crossing point of the neutral and $\sigma^{*}$ anion potential curves. ${ }^{32}$

Because the electron attachment cross section varies as $1 / E$, attachment of thermal electrons is very efficient at the stretched bond length, and signals are easily observed in spite of the relatively small number of excited molecules with energies comparable to the barrier height. The presence of low-lying $\pi^{*}$ resonances in compounds such as our chloroalkenes adds additional complexity, as the mixing between $\pi^{*}$ and $\sigma^{*}$ can shift the crossing of the adiabatic anion curve relative to the neutral. This also enhances the lifetime of the temporary anion and thus affects the survival factor. In compounds III-VI, the influence of the $\pi^{*}$ resonances at thermal electron energies has been observed in measurements by Onanong et al. ${ }^{33}$ using a commercial electron capture detector (ECD) apparatus. A signal proportional to the electron capture rate constant is observed to increase exponentially as a function of decreasing values of the VAE for attachment into the lower resonance state, reflecting the increasing involvement of the $\pi^{*}$ resonance in going from VI to III. A similar process in DNA could, in principle, lead to single strand breaks induced by electrons with thermal energies.

\section{SUMMARY}

In summary, the cross sections for remote bond breaking through the DEA process in the chloroalkenes described here can be accounted for by the fractional portion of the electron residing on the dissociating bond and the enhanced anion lifetime arising from coupling with the remote but longerlived $\pi^{*}$ resonance. The width of the composite temporary anion state can be expressed in terms of those of the unmixed resonances and the wave function amplitudes derived from their coupling. We propose that these considerations can be carried over to more complex systems such as the DNA nucleotides where they can account, in principle, for maxima in bond breaking cross sections appearing at the energies of resonances on the DNA bases.

\section{APPENDIX: A SUM RULE}

In Sec. III A 4 we pointed out that a "sum rule" is implied by our treatment of the interaction of two resonances. Although such expressions could be derived for several LASs, if the number is larger than 2, they are not expected to be very useful. Thus we continue to consider the two-state case.

The $2 \times 2$ submatrix of the Hamiltonian from Sec. III A 2 presents an example of what is known as a generalized eigenvalue problem. ${ }^{34}$ This has the general form

$$
H x=\lambda S x,
$$

where

$$
S=\left[\begin{array}{cc}
1 & S_{\pi \sigma} \\
S_{\sigma \pi} & 1
\end{array}\right]
$$

is the overlap matrix for the LAS functions $\phi_{\pi}$ and $\phi_{\sigma}$. The expressions for $\phi_{l}$ and $\bar{v}_{l E}$ corresponding to $w_{l}$ are given in Eqs. (10) and (11). The expressions for $w_{u}$ parallel to these are

$$
\phi_{u}=d_{\pi} \phi_{\pi}+d_{\sigma} \phi_{\sigma}
$$

and

$$
\bar{v}_{u E}=d_{\pi} v_{\pi E}+d_{\sigma} v_{\sigma E} .
$$

The matrix of the coefficients that give $\bar{v}_{l}$ and $\bar{v}_{u}$ may be written as

$$
U=\left[\begin{array}{ll}
c_{\pi} & d_{\pi} \\
c_{\sigma} & d_{\sigma}
\end{array}\right]
$$

where

$$
U^{\dagger} S U=I \text {. }
$$

A fairly simple transformation yields

$$
U U^{\dagger}=S^{-1},
$$

which, when expanded, implies that

$$
\begin{aligned}
c_{\pi}^{2}+d_{\pi}^{2} & =\frac{1}{\left(1-S_{\pi \sigma}^{2}\right)} \\
& =c_{\sigma}^{2}+d_{\sigma}^{2}
\end{aligned}
$$

and

$$
c_{\pi} d_{\pi}+c_{\sigma} d_{\sigma}=-\frac{S_{\pi \sigma}}{\left(1-S_{\pi \sigma}^{2}\right)} .
$$

It is now a simple substitution to see that $\Gamma_{l}+\Gamma_{u}$ is the expression given in Sec. III A 4.

${ }^{1}$ B. Boudaiffa, P. Cloutier, D. Hunting, M. A. Huels, and L. Sanche, Science 287, 1658 (2000).

${ }^{2}$ B. Boudaiffa, P. Cloutier, D. Hunting, M. A. Huels, and L. Sanche, Radiat. Res. 157, 227 (2002).

${ }^{3}$ F. Martin, P. D. Burrow, Z. Cai, P. Cloutier, D. Hunting, and L. Sanche, Phys. Rev. Lett. 93, 068101 (2004).

${ }^{4}$ R. Panajotovic, F. Martin, P. Cloutier, D. Hunting, and L. Sanche, Radiat. Res. 165, 452 (2006)

${ }^{5}$ J. Berdys, P. Skurski, and J. Simons, J. Phys. Chem. B 108, 5800 (2004); See also earlier references from these authors that are given herein.

${ }^{6}$ L. Sanche and G. J. Schulz, Phys. Rev. A 5, 1672 (1972).

${ }^{7}$ P. D. Burrow and K. D. Jordan, Chem. Phys. Lett. 36, 594 (1975).

${ }^{8}$ L. Sanche and G. J. Schulz, J. Chem. Phys. 58, 479 (1973).

${ }^{9}$ K. Aflatooni and P. D. Burrow, J. Chem. Phys. 113, 1455 (2000); This reference gives DEA measurements and a detailed analysis in a large number of chloroalkanes.

${ }^{10}$ M. Guerra, D. Jones, G. Distefano, F. Scagnolari, and A. Modelli, J. Chem. Phys. 94, 484 (1991).

${ }^{11}$ D. M. Pearl and P. D. Burrow, J. Chem. Phys. 101, 2940 (1994).

${ }^{12}$ A. Modelli, F. Scagnolari, G. Distefano, M. Guerra, and D. Jones, Chem. Phys. 145, 89 (1990).

${ }^{13}$ K. Aflatooni, G. A. Gallup, and P. D. Burrow, J. Phys. Chem. A 104, 7359 (2000). 
${ }^{14}$ In drawings like Fig. 1, one must have some care in interpreting the relative sizes of portions of an orbital. The actual coefficients in the MO show best the relative contributions of $\pi$ and $\sigma$ characters to the LUMO.

${ }^{15}$ D. M. Pearl, P. D. Burrow, J. J. Nash, H. Morrison, and K. D. Jordan, J. Am. Chem. Soc. 115, 9876 (1993).

${ }^{16}$ D. M. Pearl, P. D. Burrow, J. J. Nash, H. Morrison, D. Nachtigallova, and K. D. Jordan, J. Phys. Chem. 99, 12379 (1995).

${ }^{17}$ T. Underwood-Lemons, G. Sághi-Szabó, J. A. Tossell, and J. H. Moore, J. Chem. Phys. 105, 7896 (1996).

${ }^{18}$ Unpublished work.

${ }^{19}$ A. Modelli, J. Phys. Chem. A 109, 6193 (2005); The absolute values in this work were determined by normalization of ionization gauge reading and DEA cross sections in a series of other chlorine containing compounds.

${ }^{20}$ H. Morrison, T. V. Singh, L. de Cardenas, D. Severance, K. D. Jordan, and W. Schaefer, J. Am. Chem. Soc. 108, 3862 (1986).

${ }^{21}$ U. Fano, Phys. Rev. 124, 1866 (1961).

${ }^{22}$ A sharp-eyed reader may wonder about the units in Eq. (9), since we see from Eq. (7) that $\Gamma$ must be an energy, in spite of the fact that the Hamiltonian is squared in its definition. This arises since $\psi_{E}$ in a correct treatment has (among others) units of $E^{-1 / 2}$ and the outcome for $\Gamma$ is just the first power of energy.

${ }^{23}$ A. Messiah, Quantum Mechanics (Wiley, New York, 1966), p. $732 \mathrm{ff}$

${ }^{24}$ T. Holstein, Phys. Rev. 84, 1073 (1951).

${ }^{25}$ T. F. O’Malley, Phys. Rev. 150, 14 (1966).

${ }^{26}$ T. F. O’Malley, Phys. Rev. 156, 230 (1967).

${ }^{27}$ M. W. Schmidt, K. K. Baldridge, J. A. Boatz et al., J. Comput. Chem. 14, 1347 (1993).

${ }^{28}$ C. T. Corcoran and P. W. Langhoff, J. Math. Phys. 18, 651 (1977).

${ }^{29}$ A. U. Hazi, J. Phys. B 11, L259 (1978).

${ }^{30}$ G. A. Gallup, Valence Bond Methods (Cambridge University Press, Cambridge, 2002).

${ }^{31}$ K. Aflatooni, G. A. Gallup, and P. D. Burrow, J. Phys. Chem. A 102, 6205 (1998)

${ }^{32}$ G. A. Gallup, K. Aflatooni, and P. D. Burrow, J. Chem. Phys. 118, 2562 (2003).

${ }^{33}$ S. Onanong, P. D. Burrow, S. D. Comfort, and P. J. Shea, J. Phys. Chem. A 110, 4363 (2006).

${ }^{34}$ J. H. Wilkinson, The Algebraic Eigenvalue Problem (Oxford University Press, London, 1965), p. 337ff. 